\title{
Was gibt es Neues zum Thema invasives Neuromonitoring?
}

\author{
Update on Invasive Neuromonitoring
}

Autoren

Institut

\section{J. B. Kuramatsu, H. B. Huttner, D. Staykov}

Neurologische Klinik, Universität Erlangen-Nürnberg, Erlangen

\section{Schlüsselwörter \\ - Neuromonitoring \\ - multimodales Monitoring \\ - zerebrale Parenchymsonden \\ - Schlaganfall \\ - Schädel-Hirn-Trauma \\ Keywords \\ - neuromonitoring \\ - multimodal monitoring \\ - cerebral parenchymal probes \\ - stroke \\ traumatic-brain injury}

Bibliografie

Dol http://dx.doi.org/

10.1055/s-0035-1547268

Akt Neurol 2015; 42: 130-140

(c) Georg Thieme Verlag KG

Stuttgart - New York

ISSN 0302-4350

Korrespondenzadresse

Dr. med. Joji B. Kuramatsu

Neurologische Klinik

Universität Erlangen-Nürnberg

Schwabachanlage 6

91054 Erlangen

joji.kuramatsu@uk-erlangen.de

\section{Zusammenfassung}

$\checkmark$

Die Überwachung der strukturellen und funktionellen Integrität des Zentralnervensystems und die Vermeidung von sekundären zerebralen Schäden stehen im Mittelpunkt der neurointensivmedizinischen Behandlung. Obwohl die engmaschige klinische Untersuchung hierbei eine zentrale Rolle spielt, sind der Beurteilbarkeit schwer betroffener analgosedierter und beatmeter Patienten Grenzen gesetzt. Bildgebende Verfahren erlauben die Darstellung struktureller Schäden und einiger funktioneller Parameter wie z.B. der zerebralen Perfusion, sind jedoch nur diskontinuierlich und mit einem verhältnismäßig großen personellen und apparativen Aufwand durchführbar. Vor diesem Hintergrund wurde eine Reihe invasiver apparativer Monitoringverfahren entwickelt, die kontinuierlich oder zumindest sehr engmaschig einen Einblick in die Veränderungen des intrakraniellen Drucks, des Sauerstoffpartialdrucks im Gehirn, des zerebralen Blutflusses, oder verschiedener metabolischer Parameter erlauben. Die vorliegende Übersicht fasst die relevanten sekundären Schädigungsmechanismen in Kürze zusammen und widmet sich im Wesentlichen der Darstellung verschiedener invasiver Neuromonitoring-Verfahren inklusive der aktuellen Datenlage aus klinischen Studien. Die praktische Relevanz des invasiven Neuromonitorings wird an Hand der aktuell veröffentlichten Konsensus-Empfehlungen, der internationalen multidisziplinären Kollaboration intensivmedizinischer Fachgesellschaften, vorgestellt.

\begin{tabular}{llll}
\hline \multicolumn{2}{l}{ Abkürzungen } & MAP & Mittlerer arterieller Druck \\
$\nabla$ & & CBF & Zerebraler Blutfluss \\
SHT & Schädel-Hirn-Trauma & EVD & Externe Ventrikeldrainage \\
ICB & Intrazerebrale Blutung & TCD & Transkranieller Doppler \\
SAB & Subarachnoidale Blutung & $\mathrm{NIRS}^{2}$ & Nah-infrarot Spektroskopie \\
$\mathrm{ICP}$ & Intrakranieller Druck & $\mathrm{PtO}_{2}$ & Sauerstoffpartialdruck \\
$\mathrm{CPP}$ & Zerebraler Perfusionsdruck & $\mathrm{SD}$ & Spreading Depolarizations
\end{tabular}

\section{Abstract}

The evaluation of structural and functional integrity of the central nervous system, and prevention of secondary brain damage represents the main aim of neurocritical care. Clinical examinations are central to the management of severely injured sedated and ventilated patients, yet the value of clinical assessment is often very limited. Imaging modalities allow quantitation of structural damage and functional parameters, such a cerebral perfusion, but can only be carried out discontinuously and require great instrumental as well as personnel effort. In light of these difficulties, invasive monitoring devices have been developed, which allow continuous or at least highly frequent evaluation of changes in intracranial pressure, cerebral tissue oxygen, cerebral blood flow, and several metabolic parameters. The present review briefly summarizes the relevant mechanisms of secondary brain injury, focusing on invasive neuromonitoring techniques including available data from recent investigations. Moreover, the practical relevance of invasive neuromonitoring will be presented based on the recently published consensus statement of the international multidisciplinary collaboration of intensive care societies. 


\section{Einleitung}

Das Spektrum einer neurointensivmedizinischen Behandlung umfasst verschiedenste Erkrankungen. Der Schlaganfall mit den jeweiligen Sub-gruppen, ausgedehnte ischämische Infarkte, intrazerebrale Blutungen und subarachnoidale Blutungen stellen den größten Anteil dar. Weitere häufigere Krankheitsbilder sind das Schädel-Hirn-Trauma, der Status epilepticus, infektiöse zerebrale Erkrankungen sowie neuromuskuläre Übertragungsstörungen [1-3]. Das zentrale Ziel einer Therapie dieser Erkrankungen besteht darin, nach stattgehabtem primären Insult die drohenden Komplikationen zu verhindern, d.h. die Vermeidung von sekundären zerebralen Schäden $[2,4]$. Der kritisch kranke neurologische Patient erfordert eine hochspezialisierte Therapie aufgrund der Komplexität der zerebralen Schädigung, welche immer in direktem Zusammenhang mit den versorgenden Organen (d.h. Herz, Lunge, Niere) steht [2,4,5]. Darüber hinaus ist eine klinische Beurteilung und Überwachung dieser Patienten durch die Hirnschädigung in aller Regel nur sehr eingeschränkt möglich und somit bleibt häufig das Auftreten von sekundären Schäden inapparent $[2,4,5]$. Diese Einschränkung konfrontiert den behandelnden Arzt mit Problemen, die über das Maß einer „gewöhnlichen“ intensivmedizinischen Behandlung hinausgehen [4].

Die Neurointensivmedizin konnte sich in den letzten Dekaden als jüngste Subdisziplin innerhalb der Intensivmedizin etablieren und hat seither zu bedeutsamen Fortschritten geführt. Die Anzahl solcher Intensivstationen nimmt weltweit zu und durch diese spezialisierte Behandlung konnte eine Reduktion der Sterblichkeit und der Morbidität erreicht werden [5-7]. Trotz dieser therapeutischen Fortschritte bleibt die Sterblichkeit und der Grad der bleibenden funktionalen Behinderung weiterhin hoch $[1-3,5]$. Leider ist es bisher nur in geringem Umfang gelungen durch einzelne Interventionen, ob medikamentös, operativ oder kombiniert, einen klinischen Vorteil durch randomisiertkontrollierte Studien aufzuzeigen [1-3,5]. Diese bislang überwiegend negativen Ergebnisse deuten an, dass ein komplexes Zusammenspiel zwischen primärer Schädigung und den sich anschließenden prognose-relevanten Komplikationen besteht. Entsprechend schwierig ist es mit nur einer einzelnen (randomisiert-kontrollierten) Intervention diese umfassenden Schädigungsmechanismen zu beeinflussen $[2,4,8,9]$. Somit erscheint die Notwendigkeit einer multimodalen Behandlungsstrategie
( $\bullet$ Abb. 1) sowie eine spezialisierte als auch individualisierte Therapie dieser schwer kranken Patienten angezeigt [9].

Aktuell konnte im September 2014 ein „Consensus Statement“ zum Thema multimodales Monitoring bei kritisch kranken neurologischen bzw. neurochirurgischen Patienten veröffentlicht werden [2]. Durch die internationale multidisziplinäre Zusammenarbeit der Gesellschaften: Neurocritical Care Society (NCS), European Society of Intensive Care Medicine (ESICM), Society for Critical Care Medicine (SCCM) und des Latin America Brain Injury Consortium (LABIC) konnten Empfehlungen anhand des GRADE-Systems erarbeitet werden. Die Autoren betonen, dass dieses „Consensus Statement“ beabsichtigt, pragmatische evidenzbasierte Empfehlungen für die bettseitige Überwachung jener Patienten abzugeben, die aufgrund ihrer Hirnschädigung bzw. der notwendigen Analgosedierung einer neurologischen Untersuchung und Beurteilung nicht zugänglich sind. Es wurde ebenfalls herausgestellt, dass diese Übersicht weder eine Empfehlung zur Therapie oder bildgebenden Diagnostik noch intraoperativen Überwachung beinhalte. Sinngemäß wurde zusammengefasst: „dass kein Monitor in der Lage ist das Outcome dieser Patienten zu beeinflussen, sondern es geht vielmehr darum die zur Verfügung stehenden Daten zu interpretieren und diese in die klinische Entscheidungsfindung zu integrieren, um somit eine Therapie $z u$ optimieren welche womöglich das Outcome beeinflussen könnte. In diesem Kontext stellen die Überwachungsverfahren eine Möglichkeit dar, die pathophysiologischen Prozesse zu erforschen und potentielle neue Therapiestrategien zu identifizieren“.

Die derzeitig als Standard angewendeten Untersuchungs- und Überwachungsverfahren sind mit Limitationen behaftet [2,5]. Engmaschige klinische Untersuchungen nehmen hierbei eine zentrale Rolle ein, jedoch liefern sie nur eingeschränkte Informationen und häufig erst nachdem irreversible Schäden eingetreten sind $[2,4,5]$. Serielle Bildgebungen stellen einen größeren personellen und apparativen Aufwand dar und sind in Anbetracht schwer kranker und unter Umständen instabiler Patienten nicht ohne Risiko. Zudem liefert diese Diagnostik nur diskontinuierliche Ergebnisse. Somit erscheint die Frage berechtigt, ob mehr Daten bzw. eine kontinuierliche Erhebung den positiven Effekt der neurointensivmedizinischen Behandlung noch weiter steigern können [4-6]. Vor diesem Hintergrund wurden in den letzten Jahrzehnten invasive Verfahren entwickelt, die kontinuierlich oder sehr engmaschig Veränderungen neurophysiologischer Parameter widergeben können, welche im Folgenden vorgestellt werden $[2,4,6,10]$.

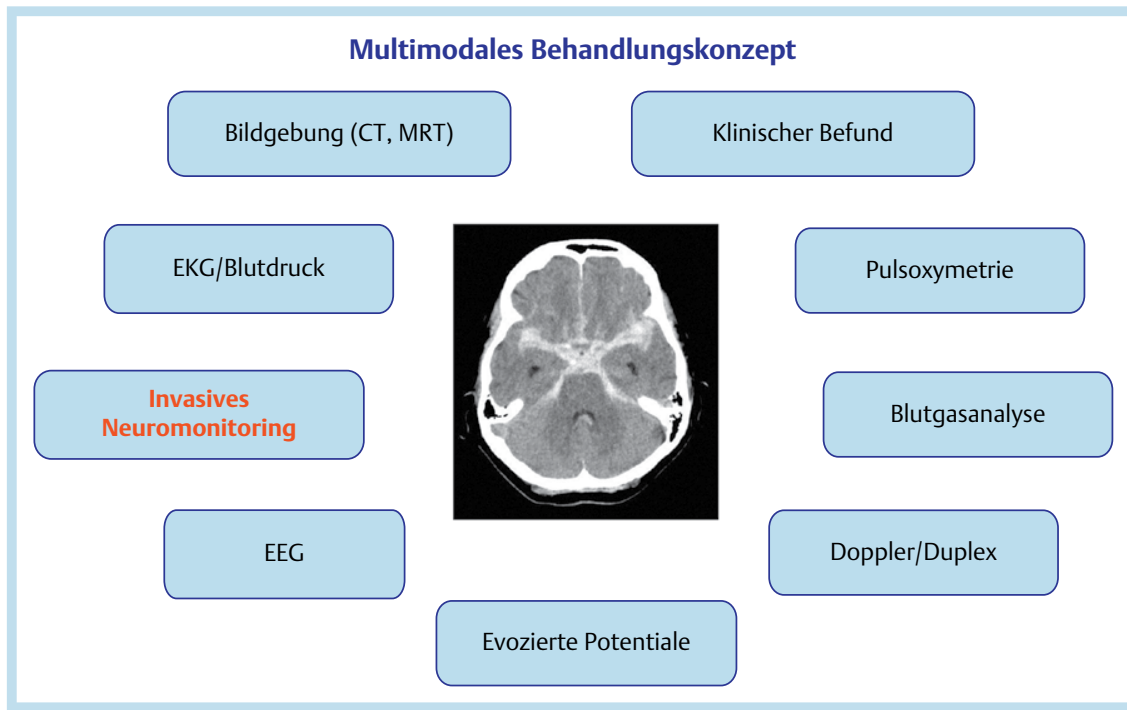

Abb. 1 Multimodales Behandlungskonzept. 


\section{Prognoseparameter und Invasive Überwachung}

Historisch betrachtet postuliert die revidierte Monroe-Kellie Doktrin, dass in dem starren Neurokranium die Summe des Volumens (Hirnparenchym, Liquor, Blut) konstant sei und das somit eine Zunahme des Einen zu einer Abnahme des Anderen führen muss. Somit müsste konzeptionell eine große intrakranielle Blutung eine Abnahme von Liquor und/oder Hirnparenchym bedingen, welches somit letztendlich einer Herniation entspräche. Dieses System beinhaltet allerdings noch weitere Kompensationsmechanismen (vermehrte venöse Drainage und Kompression des Ventrikelsystems) bevor es nach Ausschöpfung zu einem steilen intrakraniellen Druckanstieg und o.g. gefürchteten Komplikation kommt [11]. Grundsätzlich gilt es „hyperakute" Mechanismen (intrakranielle Blutung, Hämatomwachstum, akuter Hydrozephalus bzw. globales Hirnödem) welche unmittelbar (Minuten bis Stunden) nach einem primären Insult auftreten, von anschließenden sekundären Schädigungen (Minuten bis Tagen) zu unterscheiden. Im Wesentlichen wird die sekundäre Hirnschädigung durch eine Unterversorgung mit Sauerstoff und Nährstoffen (Hypoxie, Ischämie) durch verschiedene Mechanismen bedingt, z.B. Ödemprogression, zerebrale Autoregluationsstörung, Inflammation, Mikrothrombosen, „spreading depolarization“, Vasospasmen [4,12-15].

Das invasive Neuromonitoring stellt ein kontinuierliches bettseitiges Überwachungsverfahren dar, welches zur Erkennung von kritischen Hypoxien aufgrund einer Minderperfusion dienen kann. Es kann die Steuerung von Therapien und deren möglichen Nutzen unterstützen und bietet grundsätzlich einen wissenschaftlichen Mehrwert bzgl. der Erforschung von pathophysiologischen Prozessen einer sekundären Hirnschädigung als auch der Identifikation neuer Therapien $[2,4,10]$. Aktuell wird eine invasive Überwachung bei Patienten mit drohender intrakranieller Hypertension und erhöhtem Hypoxie- bzw. Infarktrisiko empfohlen. Die Indikation dafür sollte anhand einer klinischen Beurteilung oder von bildgebenden Verfahren gestellt werden. Generell werden Mikrosonden entweder über offene Kraniotomien oder Bohrlochtrepanationen in die weiße Substanz eingebracht. Diese sind im Allgemeinen mit einem niedrigen Komplikationsrisiko (1-3\%) verbunden [10,16,17]. Eine Befestigung der Sonden kann über Tunnelung oder Schraubenfixation erfolgen. Ein besonders wichtiger Punkt vor der Implantation ist die Wahl des Zielgebietes. Hier muss dem Behandler bewusst sein, dass die Messung durch eine Parenchymsonde ein loco-regionales Verfahren darstellt und keine präzise Aussage über die globale zerebrale Versorgung geben kann. Grundsätzlich besteht die Möglichkeit der Implantation der Sonde in gesunde, von der primären Läsion entfernte Areale um eine globalere Aussage zu gewinnen [10]. Die häufiger gewählte durch Experten und das Consensus Statement empfohlene Variante ist jedoch die Implantation in das Risikogebiet, d.h. in intaktes peri-läsionelles Gewebe (SHT/ICB peri-kontusionell/peri-hämorrhagisch) oder in das Gebiet mit dem höchsten Infarktrisiko (d.h. bei SAB in das Areal mit den größten Blutanteilen bzw. Stromgebiet des rupturierten Aneurysmas) [2]. Eine Implantation in die Blutung oder den Infarkt führt zu keinen sinnvollen Messungen, da Messparameter und entsprechende Schwellenwerte nur für intaktes Hirngewebe validiert sind ( $\odot$ Abb. $2-4)$.
Intrakranieller Druck und zerebraler Perfusionsdruck $\nabla$

Der intrakranielle Druck (ICP) ist der älteste Überwachungsparameter welcher herangezogen wurde, um ein Surrogat über die zerebrale Integrität zu gewinnen. Der zerebrale Perfusionsdruck (CPP) entspricht der Differenz aus mittlerem arteriellen Druck (MAP) und ICP [CPP=MAP-ICP] [11,18]. Es wird generell akzeptiert, dass ein ICP $<20-25 \mathrm{mmHg}$ angestrebt werden sollte $[2,19]$. Möglicherweise spiegelt der ICP im Sinne der MonroeKellie-Doktrin nur die Endstrecke der Prozesse einer sekundären Hirnschädigung wider und reicht häufig nicht aus um auftretende Störungen frühzeitig zu erkennen und zu therapieren [11]. Die bislang größte randomisiert-kontrollierte Studie zu SHTPatienten (BEST-TRIP Trial), welche den Nutzen einer invasiven ICP-Überwachung untersuchte, konnte keinen Vorteil eines ICP gestützten Therapiekonzepts zeigen. In dieser Studie wurden insgesamt 324 Patienten mit einem Glasgow Coma Scale Score von 3-8 eingeschlossen und das Ziel der ICP gestützten Therapie (gemessen über eine externe Ventrikeldrainage oder Parenchymsonde) war es, einen ICP kleiner $20 \mathrm{mmHg}$ aufrechtzuerhalten. Bei der Kontrollgruppe wurden nur klinische Untersuchungen und Kontrollen mittels Bildgebung durchgeführt. Beide Gruppen wurden zwar nach klar definierten studienspezifischen Leitlinien behandelt, allerdings wurden unterschiedliche Behandlungsprotokolle zur ICP-Kontrolle eingesetzt, und es wurde in der Kontrollgruppe intensiver behandelt. Somit wurde hier weniger die Effektivität einer ICP-Überwachung untersucht als der Vergleich zweier Therapieregime unternommen. Zudem wurde die Studie in Bolivien und Ecuador durchgeführt, was Anlass zu einer Debatte bzgl. prähospitalen Management als auch Anschlussheilbehandlung bot $[8,9,13]$.

Der CPP stellt einen durch MAP und ICP modifizierbaren Parameter dar und bedingt den zerebralen Blutfluss (CBF). Der Schwellenwert für einen adäquaten CPP sollte je nach Erkrankung über 50-70 mmHg liegen. Diese Grenze ist aber nur unzureichend validiert, da bisher kein Einfluss durch eine alleinige CPP-gesteuerte Therapie auf das Outcome gezeigt werden konnte. Ebenso wurden auch negative Einflüsse einer Therapie mit höheren CPP Werten beschrieben [20-24]. Darüber hinaus unterliegen CPP-Berechnungen diversen Einflussfaktoren: allen voran ist der Messwert MAP abhängig davon, ob der Druckaufnehmer auf Höhe des rechten Atriums oder des Mittelhirns platziert wird [25]. Der Druckaufnehmer sollte nach gängiger Meinung für die Berechnung des CPP auf Höhe des Foramen Monroi platziert werden, denn nur so lassen sich lagerungsbedingte Messdifferenzen vermeiden (z.B. Oberkörper auf $0-50^{\circ}$ Messdifferenz bis zu $18 \mathrm{mmHg}$ Unterschied) [2,25]. Der theoretische Nutzen einer „optimalen“ Perfusion wird zudem stark durch die zerebrale Autoregulation und die intrakranielle Compliance beeinflusst $[4,14,23,26]$.

\section{Methoden zur invasiven Messung des ICP}

Die am weitesten verbreitete Methode der ICP-Messung erfolgt durch die Implantation einer externen Ventrikeldrainage (EVD). Der abgelesene Druck entspricht dem hydrostatischen Druck des Liquor cerebrospinalis im Bereich der Spitze der EVD (meist im Vorderhorn des Seitenventrikels bzw. im Foramen Monroi). Entsprechend muss der Nullabgleich des Druckabnehmers auf einer korrespondierenden Höhe bettseitig erfolgen. Als Orientierungspunkt dient der Tragus oder der Mittelpunkt der Verbindungslinie zwischen lateralem Augenwinkel und Meatus acusticus externus. Diese Variante hat den Vorteil, akut Liquor bzw. 


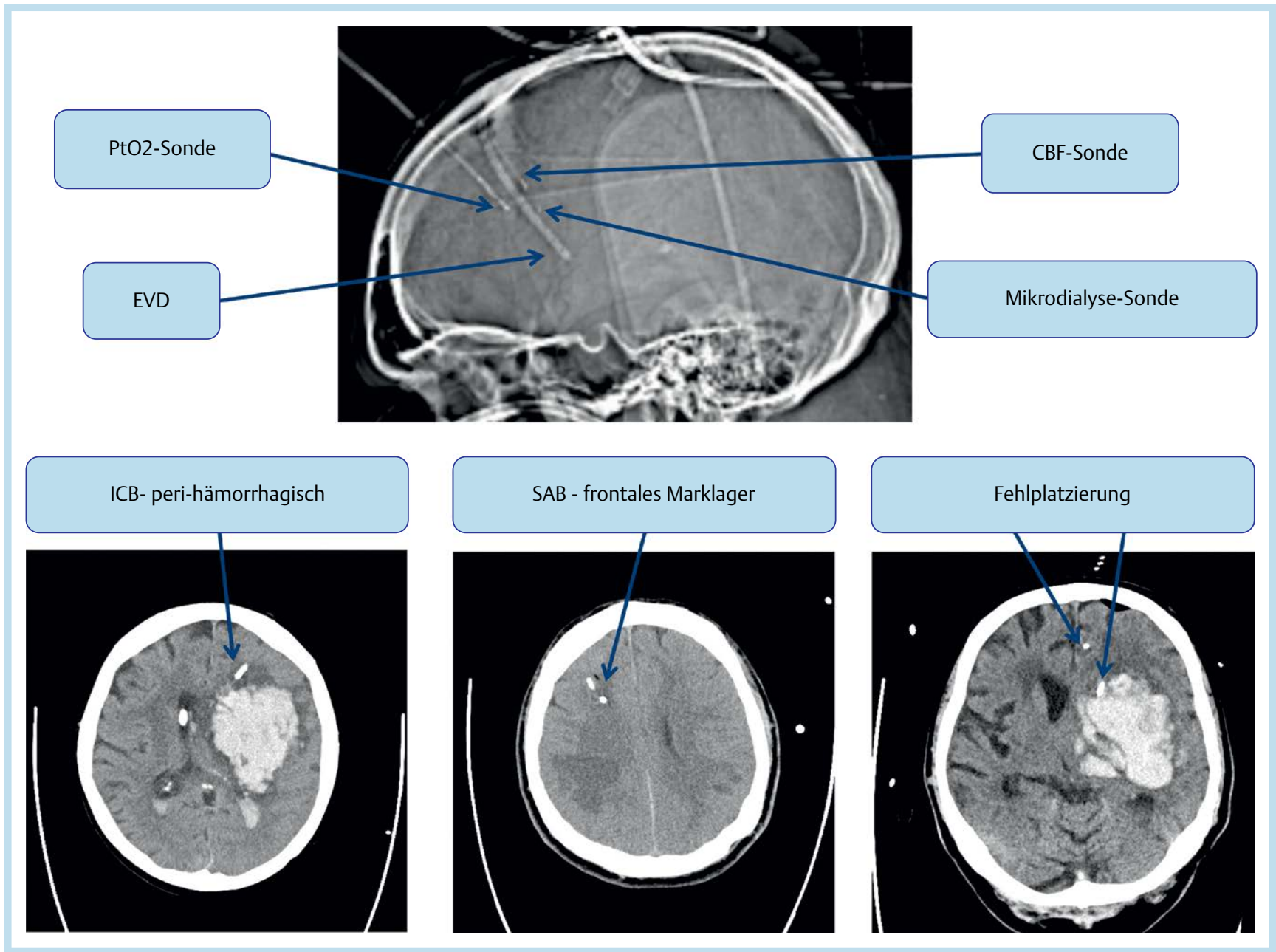

Abb. 2 Sonden Platzierung. Abkürzungen siehe Abkürzungsverzeichnis.

\begin{tabular}{|c|c|c|c|}
\hline $\begin{array}{l}\text { Sonden } \\
\text { (Anbieter) }\end{array}$ & Messparameter & Pro & Contra \\
\hline $\mathrm{CAMINO}^{\circledR}$ & ICP \& Tmp & $\begin{array}{c}\text { Langjährige Erfahrung, neue } \\
\text { Monitore }\end{array}$ & $\begin{array}{l}\text { Datenexport und } \\
\text { Kompatibilität }\end{array}$ \\
\hline HEMEDEX $^{\circledR}$ & CBF \& Tmp & $\begin{array}{l}\text { Bislang einzige } \\
\text { CPF-Sonde }\end{array}$ & $\begin{array}{l}\text { Kalibrierung (alle } 30-60 \mathrm{~min} \text { ) } \\
5 \mathrm{~min} \text { Gap, } \\
\text { Drift, Datenexport und } \\
\text { Kompatibilität }\end{array}$ \\
\hline LICOX $^{\circledR}$ & $\mathrm{Pto}^{2} \& \mathrm{Tmp}$ & $\begin{array}{c}\text { Langjährige Erfahrung, neue } \\
\text { Monitore }\end{array}$ & $\begin{array}{l}\text { Lagerung und Haltbarkeit, } \\
\text { Datenexport und } \\
\text { Kompatibilität }\end{array}$ \\
\hline MDIALYSIS ${ }^{\circledR}$ & Mikrodialyse & $\begin{array}{c}\text { MRT kompatibel } \\
\text { multiple Parameter auch } \\
\text { hochmolekulare Parameter } \\
\text { (IL, S100) }\end{array}$ & $\begin{array}{l}\text { Materialkosten, Analysator- } \\
\text { handling, regelmäßige } \\
\text { Mikrovialwechsel nötig, } \\
\text { personeller Aufwand }\end{array}$ \\
\hline NEMO $^{\circledR}$ & $\begin{array}{c}\text { ICP \& CBF \& } \\
\text { Oxygenierungstatus }\end{array}$ & $\begin{array}{l}\text { Multiparametrisch } \\
\text { Neu zugelassen, } \\
\text { Keine Erfahrung }\end{array}$ & $\begin{array}{l}\text { Neu zugelassen, } \\
\text { Keine Erfahrung }\end{array}$ \\
\hline RAUMEDIC $^{\circledR}$ & ICP \& Tmp \& Pto ${ }^{2}$ & $\begin{array}{l}\text { MRT kompatibel, } \\
\text { Einzige 3-fach Sonde }\end{array}$ & $\begin{array}{l}\text { Kunstoff-bolt, Monitor, } \\
\text { Datenexport und } \\
\text { Kompatibilität }\end{array}$ \\
\hline
\end{tabular}

Abb. 3 Invasives Monitoring (Übersicht zerebraler Parenchymsonden). (Meistgenutzte und in Deutschland erhältliche Sonden-Anbieter). Abkürzungen siehe Abkürzungsverzeichnis. 


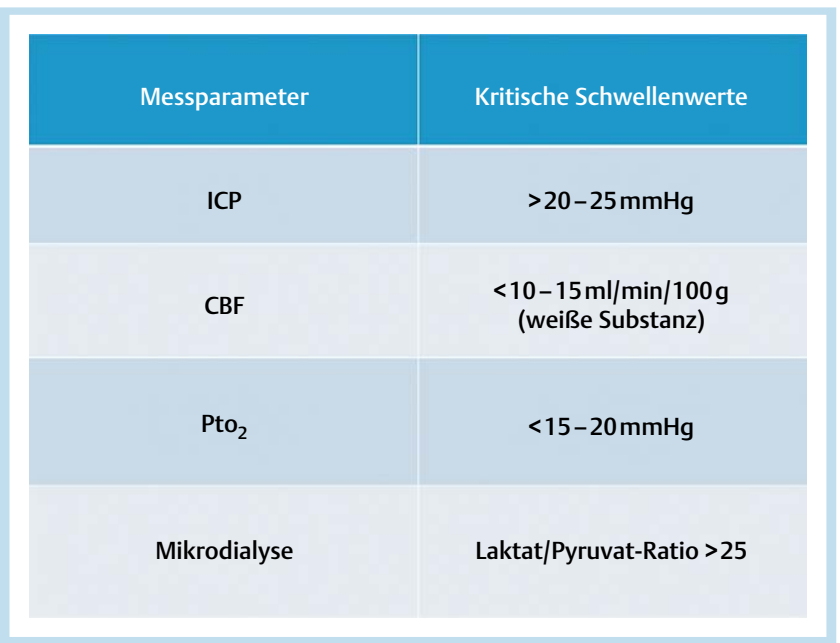

Abb. 4 Gängige Schwellenwerte der Überwachungsparameter. Abkürzungen siehe Abkürzungsverzeichnis.

Blut zu drainieren um den akuten Hydrozephalus zu behandeln, als auch die Instillation von Therapeutika zu ermöglichen [27]. Im Falle einer Ventrikelblutung mit Blockade des Foramen Monroi kann durch eine Injektion mit rtPA (intraventrikuläre Fibrinolyse) das Blutgerinnsel aufgelöst und eine adäquate Liquorzirkulation wiederhergestellt werden [27,28]. Dieses Verfahren wurde bei intrazerebralen Blutungen mit Ventrikeleinbruch erfolgreich untersucht und konnte bislang bei einem sicheren Risikoprofil positive Effekte auf das funktionelle Outcome sowie eine Reduktion der permanenten Shuntpflichtigkeit aufzeigen [29-31]. Aktuell werden Patienten im Rahmen einer internationalen randomisiert-kontrollierten Phase-III Studie zu diesem Thema rekrutiert (CLEAR III, NCT00784134), welche in naher Zukunft komplettiert sein sollte [32]. Eine weitere Option der ICP-Messung bieten Parenchymsonden als auch kombinierte EVDs mit parenchymatöser Druckmessung. Ein Vorteil dieser Methode liegt darin, z.B. bei frustraner EVD-Anlage (komprimiertes Ventrikelsystem oder bei sehr jungen Patienten mit engen Ventrikeln, bei denen die EVD oft an der Ventrikelwand anliegt und keine zuverlässige Messung des Drucks möglich ist) eine sichere ICP-Messung zu gewährleisten. Darüber hinaus ermöglicht dieses Messverfahren eine stabile kontinuierliche Messung und es können hochfrequente Werte zur Errechnung sog. Autoregulationsindices generiert werden. Ein gravierender Nachteil der ICP-Parenchymsonden ist der nach einiger Zeit auftretende Drift der Messergebnisse, welcher zu einer deutlich erschwerten Interpretierbarkeit führt (sowohl falsch hoch als auch falsch niedrig).

\section{Consensus Statement}

ICP/CPP Überwachung Patienten mit einem erhöhten Risiko für eine intrakranielle Hypertension aufgrund bildgebender oder klinischer Beurteilung sollten eine Überwachung erhalten, welche durch eine externe Ventrikeldrainage (vor allem bei akutem Hydrozephalus) oder Parenchymsonde reliabel und akkurat erfolgen kann (Wahl der Methode am besten diagnosen-spezifisch; ICB, SAB, SHT, Ventrikelblutung, Infarkte, usw.). Es sollte eine kontinuierliche Evaluation der ICP- und CPP-Werte erfolgen, dagegen sollten alleinige refraktäre ICP-Werte nicht als Prognoseparameter verwendet werden (Starke Empfehlung, moderate bis hohe Qualität der Evidenz).

\section{Zerebrale Autoregulation und Reaktivität}

$\nabla$

Die zerebrale Autoregulation gewährleistet eine konstante Durchblutung des Gehirns bei Blutdruckschwankungen. Sie wird durch den basalen Tonus der arteriellen Gefäßmuskulatur der Hirnarterien erreicht und bleibt über einen sehr weiten MAPDruckbereich (für Normotoniker: $50-150 \mathrm{mmHg}$ ) konstant [18, $26,33,34]$. Man spricht von einer gestörten Autoregulation, wenn das Gefäßsystem sich passiv gegenüber Druckschwankungen verhält; z.B. eine Erhöhung des MAP würde zu einer passiven Dehnung bzw. Dilation der Gefäße mit einer konsekutiven intrakraniellen Volumenbelastung und somit intrakraniellem Druckanstieg führen. Neben der Druckregulation existieren auch metabolische Regulationsmechanismen, bspw. können Elektrolytverschiebungen (Erhöhung von Kalium) oder auch eine Zunahme von Laktat über eine Azidose zu einer Vasodilatation führen [35]. Die chemische Autoregulation stellt eine weitere wesentliche Stellgröße der CPP-Regulierung dar, welche über Veränderungen des $\mathrm{CO}_{2}$-Partialdrucks zu einer Veränderung der Durchblutung und Volumenbelastung führen kann [18,34-36]. Diese Effekte können auch prinzipiell therapeutisch genutzt werden, z.B. die kontrollierte Hyperventilation, welche über die Induktion einer Hypokapnie und Alkalose zu einer Abnahme des ICP führt. Allerdings sollte eine Hyperventilation nur als allerletzte Maßnahme und v. a. kurzzeitig ( $<30 \mathrm{~min}$ ) durchgeführt werden. Die ICP-Senkung wird hier durch eine Vasokonstriktion und Abnahme des zerebralen Blutvolumens erreicht aber durch Adaptionsmechanismen bei längerfristiger Hyperventilation (Vermeidung von einem $\mathrm{PaCO}_{2}<30 \mathrm{mmHg}$ ) wird der Nutzen abgeschwächt bzw. kann die Behandlung sogar schädlich sein (Rebound-Phänomen) $[37,38]$. Ebenfalls wird der Einfluss von Sedativa und Analgetika mit potentiell negativen Effekten auf den CPP diskutiert; vermehrte bzw. prolongierte Dosen können zu einer Reduktion der zerebralen Durchblutung führen [36]. Zunehmend werden zur Quantifizierung einer intakten bzw. gestörten Autoregulation sogenannte Reaktivitätsindices herangezogen. Diese beruhen in aller Regel auf einer Korrelationsanalyse des ICP mit anderen blutflussassoziierten Monitoringparametern (z.B. MAP, TCD, Sauerstoffpartialdruck, NIRS) [34,39-44]. Es konnte in diversen monozentrischen Studien an Patienten mit SHT, SAB und ICB gezeigt werden, dass eine gestörte Autoregulation mit sekundären Ischämien und mit schlechterem funktionellen Outcome assoziiert ist [39-44]. Es wurde hierbei ein sog. „optimaler“ CPP (СРPopt) mithilfe eines Autoregulationsindex (PRx) berechnet. Prognose relevante Vorteile ergaben sich für die Patienten, deren reale CPP-Werte am besten mit dem CPPopt korrelierten [39-44]. Eine kürzlich veröffentlichte Meta-Analyse zu diesem Thema hebt die Relevanz und Wichtigkeit dieser Reaktivitätsindices als Parameter im Rahmen einer individualisierten CPP gesteuerten Therapie hervor. Diese Indices sollten hinzugezogen werden um eine bessere Beurteilung der zerebralen Sauerstoffversorgung und des metabolischen Status des Gehirns zu gewährleisten [45]. In diesem Sinne stellt der „optimale“ CPP keine starre Größe dar, sondern möglichweise sind unterschiedliche CPP-Werte in Abhängigkeit der zerebralen Autoregulation notwendig um eine adäquate Durchblutung zu erzielen.

\section{Consensus Statement}

Überwachung der zerebralen Autoregulation Eine kontinuierliche Überwachung der Autoregulation ist möglich und sollte in Erwägung gezogen werden bei ähnlicher Validität der verschiedenen Verfahren. Die Evaluation und Überwachung der 
Autoregulation könnte nützlich sein zur Erreichung einer optimalen zerebralen Perfusion und auch hilfreich zur Abschätzung der Prognose nach akuter zerebraler Schädigung (Schwache Empfehlung, moderate Qualität der Evidenz).

\section{Methoden zur invasiven zerebralen Blutflussmessung} Seit knapp 10 Jahren steht ein Verfahren der direkten regionalen Blutflussmessung zur Verfügung. Der regionale Blutfluss wird über ein Thermodiffusionsverfahren errechnet, welches den Blutfluss über den konvektiven Wärmeabtransport zwischen 2 Thermistoren, die in der Spitze einer Parenchymsonde integriert sind, ermittelt. Die Messung umfasst ein Gewebevolumen von ca. $5 \mathrm{ml}$ um die Sondenspitze [46]. Dieses Verfahren wurde gegenüber der quantitativen CBF-Errechnung mittels Xenon-CT etabliert und liefert Werte mit guter Korrelation. Allerdings sind bei dieser Parenchymsonde in Intervallen (30-60 min) Rekalibrierungen notwendig, welche zu ca. 5 minütigen Messpausen führen. Zudem zeigen neuere Daten, dass ein nicht zu vernachlässigender Drift existiert, welcher potentiell zu einer möglichen Überschätzung der Messwerte führen könnte [46].

Ein völlig neues Messverfahren zur CBF-Messung hat aktuell die Zertifizierung absolviert und voraussichtlich 2015 soll eine kombinierte Mikrosonde auf den Markt kommen [47,48]. Diese Sonde ist mit optischen Fasern (near infrared spectroscopy) bestückt, welche Licht emittieren und anhand der Reflexion von unterschiedlichen gewebeabhängigen Absorptionsspektren folgende Parameter analysiert. Der CBF wird berechnet über die spektroskopische Messung der Dilution von intravenös appliziertem Indocynanid-Grün, dem zerebralen Blutvolumen und der durchschnittlichen mittleren Transitzeit. Diese multiparametrische Sonde misst des Weiteren den ICP, die Temperatur und den Oxygenierungsstatus von Hämoglobin (oxygeniertes bzw. desoxygeniertes Hämoglobin). Diese Methode wurde bislang nur an wenigen Patienten untersucht, lieferte aber konsistente und mit gängigen Methoden vergleichbare Werte bei einem ähnlichem Risikoprofil $[47,48]$. Auch dieses Verfahren ist loco-regional auf ein gewisses Hirnparenchymvolumen begrenzt. Es besteht eine mögliche Indikation zur CBF-Messung vor allem bei Patienten mit aneurysmatischen SAB und SHT aufgrund des erhöhten Ischämierisikos (z. B. bei Vasospasmen). Insgesamt hat trotz langjähriger Erfahrung die zerebrale Blutflussmessung jedoch noch wenig Einzug in die klinische Routine gehalten und die Fallzahlen der Patienten innerhalb der publizierten Studien sind nicht ausreichend.

\section{Consensus Statement}

Überwachung des zerebralen Blutflusses Eine CBF-Sonde kann zur Detektion von Patienten mit fokalem Ischämierisiko im Erfassungsgebiet der Sonde hilfreich sein und sollte bei Patienten mit einer aneurysmatischen SAB in das Stromgebiet des rupturierten Aneurysmas platziert werden (Schwache Empfehlung, sehr niedrige Qualität der Evidenz).

\section{Zerebraler Gewebesauerstoff (Sauerstoffpartialdruck, $\mathrm{PtO}_{2}$ ) \\ $\nabla$}

Die invasive Messung des Sauerstoffpartialdrucks stellt ein weiteres regionales Verfahren dar, welches Rückschluss auf den zerebralen Blutfluss, Oxygenierung und potentielles Hypoxierisiko zulässt [49]. Es konnte bei SHT- und SAB-Patienten gezeigt werden, dass sekundäre hypoxische Episoden unerkannt blie- ben, wenn sich die Überwachung ausschließlich auf ICP/CPP stützte, obgleich normale Messwerte vorlagen [50,51]. Aktuell befindet sich eine randomisiert-kontrollierte Studie in der Finalisierungsphase, welche den zusätzlichen Nutzen einer $\mathrm{PtO}_{2}$ gestützten Therapie bei SHT-Patienten untersucht. Vielversprechende Vorabergebnisse dieser Phase-II Studie wurden auf der Neurocritical Care Conference 2014 präsentiert und zeigten eine reduzierte Hypoxiedauer, eine reduzierte Mortalität und einen positiven Einfluss auf das funktionelle Outcome (BOOST II; NCT00974259). Ähnliche Effekte mit einer Verbesserung des funktionellen Outcomes, durch eine $\mathrm{PtO}_{2}$ gestützte Therapie bei SHT-Patienten, wurden in einer kürzlich veröffentlichten MetaAnalyse gezeigt [52]. Darüber hinaus konnte eine monozentrische Untersuchung auch den Vorteil einer $\mathrm{PtO}_{2}$ gestützten Behandlung bei SAB-Patienten unterstreichen [53]. Auch können Interventionen überwacht bzw. kontrolliert werden; z.B. CPPSteuerung, Beatmung, Sedierung und Effekte von Bluttransfusionen [54]. Wie bereits oben erwähnt, wird zunehmend eine Kombination aus ICP- und $\mathrm{PtO}_{2}$-Messung zur Beurteilung der Autoregulation (ORx) eingesetzt, welche bereits positive Einflüsse auf klinische Endpunkte in prospektiven Untersuchungen zeigen konnte $[39,45,53,55]$.

\section{Methoden der invasiven}

\section{Sauerstoffpartialdruckmessung im Gehirn}

Aktuell stehen 2 unterschiedliche Mikrosonden zur Verfügung. Die eine Sonde nutzt ein biochemisches Verfahren nach dem Clark-Prinzip zur Messung der Sauerstoffkonzentration, die andere ein photooptisches Verfahren mittels FluoreszenzlichtMessung (quenching of luminescence). Erstere stellt die ältere und somit bislang meist genutzte Sonde dar, auf welcher der überwiegende Anteil der klinischen Untersuchungen basiert. Aktuell wurde das Monitorsystem für diese Sonde überarbeitet, welches noch in diesem Jahr auf den Markt kommen und dem Anwender einen eigenständigen bettseitigen Monitor bieten soll. Die neuere Variante wird als eine kombinierte Sonde angeboten, die über den $\mathrm{PtO}_{2}$ hinaus zusätzlich ICP und Temperatur messen kann. Es gilt zu erwähnen, dass der in-vivo Vergleich beider Systeme bei parallel einliegenden Sonden zu signifikant unterschiedlichen Messwerten geführt hat, jedoch ist der Grund hierfür bislang ungeklärt [56]. Grundsätzlich hat sich aber die $\mathrm{PtO}_{2-}$ Messung aufgrund stabiler Datenqualität und geringer Artefaktanfälligkeit etabliert.

\section{Consensus Statement}

Überwachung des zerebralen Gewebesauerstoffs Eine Überwachung sollte in Erwägung gezogen werden bei Patienten mit einem erhöhten Hypoxie- bzw. Ischämierisiko mittels Parenchymsonde und/oder jugulär-venöser Oxymetrie. In Abhängigkeit von der zugrundeliegenden Pathologie und technischen Machbarkeit sollte die Lokalisation der Sonde bzw. des Katheters gewählt werden. Eine akkurate Prognoseabschätzung sollte immer in Konjugation mit weiteren klinischen oder anderen Überwachungsmodalitäten vollzogen werden (Starke Empfehlung, niedrige Qualität der Evidenz).

\section{Spreading Depolarizations}

Ein elektrisches Phänomen welches seit Jahrzehnten aus der Epileptologie und Migräne-Forschung bekannt ist, aber erst seit kurzem ein reges wissenschaftliches Interesse bei kritisch kran- 
ken neurologischen Patienten geweckt hat, sind die sog. „spreading depolarizations“ (SD) $[15,57]$. Die internationale COSBID (Cooperative Study of Brain Injury Depolarizations) Forschungsgruppe untersucht diese Thematik experimentell seit einigen Jahren mit außergewöhnlichem Aufwand (Elektrokortikografie und zusätzliche diverse parenchymale Sonden) und konnte hierbei neue Erkenntnisse bzgl. der pathophysiologischen Prozesse der sekundären zerebralen Schädigungsmeschanismen gewinnen $[12,15,58,59]$. Bei Patienten mit akuter zerebraler Schädigung können SD in gesundem Hirngewebe entstehen und zu einer metabolischen Einschränkung führen, aber führen dort nicht zwangsläufig zu einer Ischämie bzw. irreversiblen neuronalen Schädigung. Allerdings können sich in peri-läsionellen Arealen bzw. metabolisch derangiertem Gewebe diese fortlaufenden, propagierenden, depolarisierenden Wellen (peri-infarct depolarizations) aggravierend auswirken, sich ausdehnen und um die Läsion kreisen [12,15,58-60]. Entsprechend können SD eine Störung der neuronalen Homöostase mit erhöhter hypoxischer Vulnerabilität und reduziertem Blutfluss bewirken, welche schließlich zu einem Zelluntergang führen könnte. Dieser wiederum könnte sich auch auf das funktionelle Outcome übertragen [60]. Bei Patienten mit SHT, malignen Mediainfarkten, SAB und ICB konnten SD nachgewiesen werden und es konnte teilweise ein unabhängiger Einfluss der SD auf das Outcome festgestellt werden. Darüber hinaus konnten theoretische Zusammenhänge zwischen der Hypoxie und Infarzierung sowie der möglicherweise im Vorfeld ablaufenden Prozesse (gestörte Ionen-Homöostase, Ausschüttung exzitatorischer Aminosäuren, Hyper- bzw, Hypoperfusion, gestörte Autoregulation, inverses neurovaskuläres Coupling) aufgezeigt werden [12,15,58-61]. Hierdurch konnte erstmals eine Art theoretischer Zusammenhang der komplexen Mechanismen einer sekundären Hirnschädigung formuliert werden: die zerebrale Perfusion (Blutfluss) als Summe seiner Faktoren, insbesondere der wechselseitige Einfluss von zerebraler Autoregulation und Sauerstoffversorgung sowie die dadurch bedingten Auswirkungen auf die metabolische Stoffwechselsituation (bzw. metabolische Vulnerabilität) unter dem Einfluss von SD; letztlich allesamt Stellgrößen die zusammen mögliche Infarzierungen bedingen könnten $[12,15$, 58-60].

\section{Entzündungsreaktion und Mikrothrombosen \\ $\nabla$}

Dieses Feld wird bislang immer noch durch die tierexperimentelle Forschung dominiert, allerdings werden zunehmend Liquoranalysen und Mikrodialyseverfahren in vivo angewendet, um die pathophysiologischen Assoziationen von Cytokinen, Chemokinen, Metalloproteinasen und Nekrosefaktoren nach stattgehabter SAB oder nach SHT zu untersuchen. Überwiegend wurde der Einfluss auf die Genese von angiografisch nachgewiesenen Vasospasmen evaluiert, jedoch konnten hier keine kongruenten Ergebnisse gezeigt werden [62-64]. In kürzlich veröffentlichten Meta-Analysen konnten verschiedene Biomarkern identifiziert werden, die eine Korrelation mit der Prognose nach SAB bzw. SHT aufzeigen, ohne dass diese eine sichere Prädiktion leisten könnte (d.h. IL-6, IL-1ß, ET-1, TNF-alpha usw.) [62-64]. Subarachnoidale Blutanteile können einerseits zu einem vermehrten „scavenging“ von vasodilatatorisch wirksamen NO führen und andererseits kann vermehrt ungebundenes Eisen zu erhöhtem oxidativen Stress und konsekutiven Vasospasmen bzw. Mikrozirkulationsstörungen führen $[14,15,34]$. Autopsiestudien weisen darauf hin, dass Mikrothrombosen in Arealen mit sekundärer Infarzierung ebenfalls in großem Umfang vorliegen und somit in ätiologischem Zusammenhang mit sekundären Infarkten stehen könnten [65-67]. Klinische Untersuchungen zu diesem Phänomen sind kaum realisierbar, und post-hoc Analysen aus Studien zu Aggregationshemmern bei SAB zeigen keinen Effekt auf das klinische Outcome [14].

\section{Neurochemische Messungen metabolischer Parameter} mittels Mikrodialyse

Die zerebrale Mikrodialyse stellt ein loco-regionales Verfahren dar um die Stoffwechselsituation in gewissen (meist stündlichen) Messintervallen abzubilden. Zu diesem Zweck wird ein in der Parenchymsonde integrierter Doppellumen-Mikrokatheter mit einer semipermeablen Membran ( $25 \mathrm{kD}$ ) von einer isotonen Perfusionflüssigkeit umspült. So können zu analysierende Metabolite (Glukose, Laktat, Pyruvat, Glutamat, Glycerol) aus dem Extrazellulärraum entlang des Gradienten in das Mikrodialysat hinein diffundieren $[68,69]$. Dieses wird über das Pumpensystem in ein Gefäß (microvial) aufgenommen und kann anschließend bettseitig analysiert werden $[68,69]$. Ferner steht auch ein Katheter mit einer höher-molekularen Membran zur Verfügung (100 kD), der es ermöglicht, entsprechende Moleküle (z.B. Interleukine, S100) und Pharmaka zu untersuchen [70,71]. Etabliert wurde die Methode initial überwiegend an SHT- und SABPatienten und es konnte aufgezeigt werden, dass ein Glukoseabfall, unabhängig vom Blutglukosespiegel, in Kombination mit einem Anstieg des Verhältnisses von Laktat und Pyruvat (Laktat/ Pyruvat-Ratio) mit einer hypoxischen Stoffwechselsituation bzw. mitochondrialen Dysfunktion einhergeht. Für diese Konstellation konnten folgende prädiktive Assoziationen gezeigt werden: bei SAB-Patienten war eine erhöhte Laktat/PyruvatRatio mit dem Auftreten von verzögerten ischämischen Defiziten (delayed ischemic neurological deficits) und Vasospasmen assoziiert. Bei SHT-Patienten korrelierte dies mit einer zerebralen Hypoxie und schlechterem funktionellen Outcome sowie mit ICP-Krisen [68, 72, 73]. Die bislang größte Studie an 223 SHTPatienten konnte darüber hinaus eine erhöhte Laktat/PyruvatRatio als unabhängigen Prädiktor für Outcome und Mortalität ableiten und eine klinisch relevante Phase (innerhalb der ersten $72 \mathrm{~h}$ nach Ictus) mit erhöhter metabolischer Vulnerabilität identifizieren. Eine neuere, allerdings noch kaum verbreitete Methode (bislang nicht kommerziell erhältlich) kommt ohne händisches Wechseln der „microvials“ aus; über ein automatisiertes Verfahren (rapid-sampling) können hochauflösend (Sekunden bis Minuten) Messwerte gewonnen werden. Die zerebrale Mikrodialyse wird seit Ende der 90er Jahren eingesetzt und gilt als ein sicheres und etabliertes Verfahren.

\section{Consensus Statement}

Überwachung des zerebralen Stoffwechsels Eine MikrodialyseSonde sollte bei Patienten mit erhöhtem Ischämie- bzw. Hypoxie-risiko und Stoffwechselversagen sowie Glukosemangel in Erwägung gezogen werden. Die Platzierung der Sonde sollte in Erwägung der zugrundeliegenden Pathologie und technischen Machbarkeit erfolgen. Obwohl dauerhaft erniedrigte zerebrale Glukosewerte und ein erhöhtes Laktat-Pyruvat Verhältnis prädiktive Parameter einer erhöhten Sterblichkeit und einer funktionellen Beeinträchtigung sind, sollten trotzdem immer weitere klinische oder andere Überwachungsmodalitäten für eine akkurate Prognoseabschätzung in Betracht gezogen werden (Starke Empfehlung, niedrige Qualität der Evidenz). 


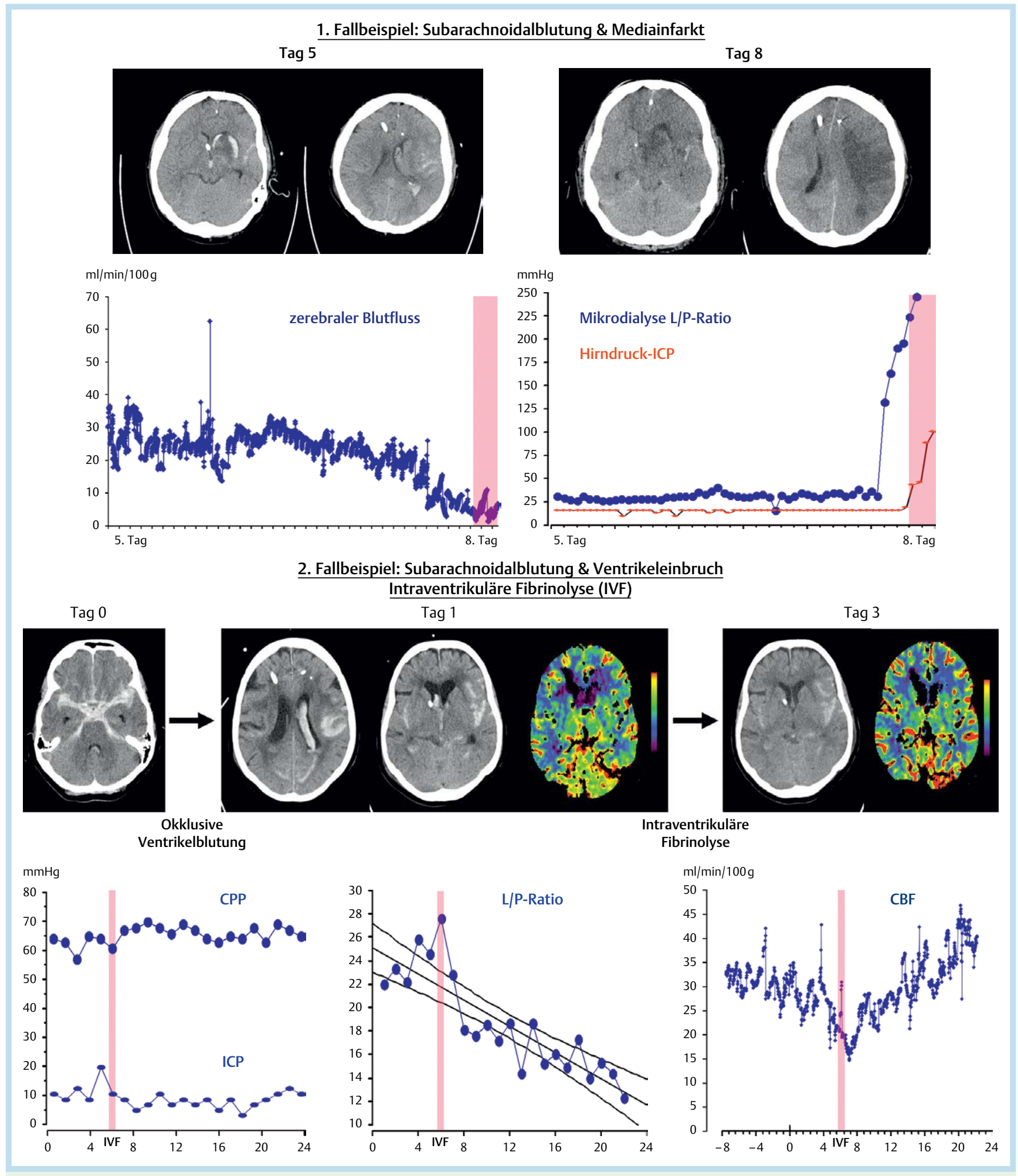

Abb. 5 Fallbeispiele.

\section{Fallbeispiele (० Abb. 5)}

$\nabla$

1. Fall: 53-jähriger Patient mit einer SAB (Hunt\&Hess ${ }^{\circ} 4$ ) bei Arteria communicans- Aneurysma, parenchymatösen Blutanteilen und einer intraventrikulären Blutung. Initial erfolgte bei einer Liquorzirkulationsstörung die Anlage einer externen Ventrikeldrainage sowie eine interventionelle Versorgung des Aneurysmas. Am Folgetag wurden in das links fron- tale Marklager Parenchymsonden (CBF und Mikrodialyse) implantiert. Der Patient erhielt bei kritischen ICP-Anstiegen eine maximale antiödematöse und eine intravenöse antivasospastische Therapie. Im Verlauf zeigten sich im transkraniellen Doppler ab Tag 5 leicht progrediente Flussgeschwindigkeiten (Lindegaard-Ratio <3). In der Nacht von Tag 7 auf 8 kam es zu einer kritischen Abnahme des CBF sowie einem rasanten Anstieg des Laktat/Pyruvat Verhältnisses bei weiter- 
hin stabiler ICP-Situation. In der folgenden Bildgebung stellte sich eine neu aufgetretene Infarktdemarkation im kompletten Mediastromgebiet links mit beginnender transfalxialer und transtentorieller Herniation dar. Der Patient verstarb kurz darauf (Tag 8). Fazit-PRO: Messparameter zeigten kritische Veränderungen der zerebralen Versorgung 6-8 Stunden vor der ICP-Entgleisung. Contra: Für die Platzierung der Sonden wurde das Stromgebiet der A. cerebri anterior bzw. das Grenzstromgebiet gewählt, allerdings wurde somit die aufgetretene Hypoxie im MCA-Territorium nicht erfasst und entsprechend konnte keine präventive Therapie eingeleitet werden um eine Infarzierung zu verhindern.

2. Fall: Eine 78-jährige Patientin mit einer SAB (Hunt\&Hess ${ }^{\circ} 3$ ) bei linksseitigem Aneurysma der MCA mit intraventrikulären Blutanteilen wurde initial endovaskulär versorgt und erhielt bei beginnender Liquorzirkulationsstörung eine externe Ventrikeldrainage. Bei ausgeprägtem bildmorphologischem Befund mit Bluttamponade der Zisternen (modified Fisher Scale von 4; ein Wert von 0 bedeutet keine subarachnoidalen od. intraventrikulären Blutanteile bis hin zu einem maximalen Wert von 4, welcher eine ausgedehnte „dicke“ subarachnoidale Blutung mit intraventrikulären Blutanteilen bedeutet) wurde ein invasives Monitoring mittels Parenchymsonden (CBF und Mikrodialyse) indiziert. Innerhalb von 12 Stunden nach Sondenanlage zeigte sich eine Abnahme des zerebralen Blutflusses und ein Anstieg des Laktat/Pyruvat Verhältnisses, ohne dass eine ICP/CPP Überwachung wesentliche kritische Werte bot. Die anschließende Bildgebung ergab eine Blockade des Foramen Monroi mit akuter Liquorzirkulationsstörung. Bei versorgtem Aneurysma wurde eine intraventrikuläre Fibrinolysetherapie mit rtPA durchgeführt, woraufhin sich die Monitoringparameter innerhalb von wenigem Stunden signifikant verbesserten. Der weitere stationäre Verlauf gestaltete sich komplikationslos und im 3-Monate-Follow-up war die Patientin selbstständig mobil und hatte nur noch geringe Funktionseinschränkungen (mRS 2). Fazit-PRO: Die invasive multimodale Überwachung zeigte frühzeitig eine Einschränkung der globalen zerebralen Versorgungssituation bei neu aufgetretenem okklusivem Hydrozephalus an. Es konnte somit eine Therapie (intraventrikuläre Fibrinolyse) vor dem Auftreten von sekundären Schädigungen eingeleitet werden und eine Restitution der zerebralen Versorgungsituation adäquat überwacht werden.

\section{Fazit für die Praxis}

Der mögliche Nutzen und potentielle klinische Vorteil eines invasiven multimodalen Neuromonitorings wird durch die überwiegend starke Empfehlungskategorie innerhalb dieses Consensus Statements ganz aktuell hervorgehoben. Allerdings ist abgesehen von einer ICP- und CPP-Überwachung, trotz der hohen Empfehlungsstärken die Qualität der Evidenz für die weiteren Modalitäten nur moderat bis sehr niedrig. Somit entsprechen diese Empfehlungen im Wesentlichen Expertenmeinungen und von einer unkritischen Anwendung des invasiven Neuromonitoring muss abgeraten werden. Bislang fehlt eine Validierung eines solchen multimodalen Therapiekonzeptes durch randomisiert-kontrollierte Studien, allerdings nimmt der wissenschaftliche Wissenszuwachs der durch diese Verfahren erzielt worden ist einen immens wichtigen Stellenwert ein. Des Weiteren gilt es einige Faktoren, die in unmittelbarem Zusammenhang mit der Durchführbarkeit einer invasiven multimodalen Behandlung stehen, kritisch zu hinterfragen. Vorrangig ist eine solche Strategie nur mit einem hohen logistischen Aufwand möglich (multidisziplinäres Team mit hoher zeitlicher Valenz), das Behandlungskonzept ist sehr kostenintensiv und ein hohes Maß an pflegerischer Kapazität, Schulung und Compliance muss voraussetzt werden. Ein komplexeres invasives Monitoring ist nur dann sinnvoll, wenn die Sonden adäquat platziert sind, und vor allem auch über den kritischen Überwachungszeitraum an Ort und Stelle bleiben. Essentiell ist, dass die Hardware und die Software bedienbar und leicht verständlich sind und eine intermodale Kompatibilität einfach herstellbar ist, d.h. „plug and play“ und das verschiedene Systeme leicht zu kombinieren sind und vor allem hochaufgelöste, kontinuierliche Daten zentral erfasst und abgebildet werden können. Dieser Punkt obliegt den Anbietern und stellt den Anwender häufig noch vor große Hürden. Eine weitere Hürde in einem solchen Setting ist womöglich der behandelnde Arzt selbst. Hiermit ist nicht eine mangelnde fachliche Kompetenz, sondern die menschliche Aufnahmefähigkeit gemeint. Eine Flut von verschiedenen Parametern trägt nicht dazu bei, eine rasche Integration dieser Datenfülle und der konsekutiven Interpretation in Hinblick auf die klinische Relevanz zu erleichtern. Womöglich erschwert dies sogar in der Akutsituation das Fällen einer schnellen therapeutischen Entscheidung. Darüber hinaus bestehen weiterhin berechtigte Einwände gegenüber der Validität dieser gewonnen Parameter und deren prognostischen Bedeutung. Die Hoffnung besteht allerdings, dass zukünftig mit besserer Studienlage und zunehmender Integration von bioinformatischer Verfahren die kritische Entscheidungsfindung erleichtert werden kann, um so dem Patienten die bestmögliche Therapie bei größtmöglicher Sicherheit zu bieten [4].

\section{Interessenkonflikt}

$\nabla$

Die Autoren geben an, dass kein Interessenkonflikt besteht.

\section{Literatur}

1 Dagal A, Lam AM. Cerebral blood flow and the injured brain: how should we monitor and manipulate it? Curr Opin Anaesthesiol 2011; 24: $131-137$

2 Le Roux P, Menon DK, Citerio $G$ et al. Consensus summary statement of the International Multidisciplinary Consensus Conference on Multimodality Monitoring in Neurocritical Care: a statement for healthcare professionals from the Neurocritical Care Society and the European Society of Intensive Care Medicine. Intensive Care Med 2014; 40: 1189-1209

3 Le Roux PD, Cooper J, Guntupalli KK et al. The critical care research networks experience. Neurocrit Care 2012; 16: 20-28

4 Hemphill JC, Andrews P, De Georgia M. Multimodal monitoring and neurocritical care bioinformatics. Nat Rev Neurol 2011; 7: 451-460

5 Rincon F, Mayer SA. Neurocritical care: a distinct discipline? Curr Opin Crit Care 2007; 13: 115-121

6 Korbakis G, Bleck T. The Evolution of Neurocritical Care. Crit Care Clin 2014; 30: 657-671

7 Suarez JI. Outcome in neurocritical care: advances in monitoring and treatment and effect of a specialized neurocritical care team. Crit Care Med 2006; 34: S232-S238

8 Sarrafzadeh AS, Smoll NR, Unterberg AW. Lessons from the intracranial pressure-monitoring trial in patients with traumatic brain injury. World Neurosurg 2014; 82: e393-e395

9 Le Roux P. Intracranial pressure after the BEST TRIP trial: a call for more monitoring. Curr Opin Crit Care 2014; 20: 141-147

10 Diedler J, Czosnyka M. Merits and pitfalls of multimodality brain monitoring. Neurocrit Care 2010; 12: 313-316 
11 Mokri B. The Monro-Kellie hypothesis: applications in CSF volume depletion. Neurology 2001; 56: 1746-1748

12 Hartings JA, Wilson JA, Hinzman JM et al. Spreading depression in continuous electroencephalography of brain trauma. Ann Neurol 2014; 76: 681-694

13 Stocchetti $N$, Le Roux P, Vespa P et al. Clinical review: neuromonitoring - an update. Crit Care 2013; 17: 201

14 Macdonald $R L$. Delayed neurological deterioration after subarachnoid haemorrhage. Nat Rev Neurol 2014; 10: 44-58

15 Dreier JP. The role of spreading depression, spreading depolarization and spreading ischemia in neurological disease. Nat Med 2011; 17 : 439-447

16 Munch E, Weigel $R$, Schmiedek $P$ et al. The Camino intracranial pressure device in clinical practice: reliability, handling characteristics and complications. Acta Neurochir (Wien) 1998; 140: 1113-1119 discussion 1119-1120

17 Stuart RM, Schmidt M, Kurtz $P$ et al. Intracranial multimodal monitoring for acute brain injury: a single institution review of current practices. Neurocrit Care 2010; 12: 188-198

18 Kirkman MA, Smith M. Intracranial pressure monitoring, cerebral perfusion pressure estimation, and ICP/CPP-guided therapy: a standard of care or optional extra after brain injury? Br J Anaesth 2014; 112: 35-46

19 Resnick DK, Marion DW, Carlier P. Outcome analysis of patients with severe head injuries and prolonged intracranial hypertension. J Trauma 1997; 42: 1108-1111

20 Dizdarevic K, Hamdan A, Omerhodzic I et al. Modified Lund concept versus cerebral perfusion pressure-targeted therapy: a randomised controlled study in patients with secondary brain ischaemia. Clin Neurol Neurosurg 2012; 114: 142-148

21 Muzevic D, Splavski B. The Lund concept for severe traumatic brain injury. Cochrane Database Syst Rev 2013; 12: CD010193

22 Bratton SL, Chestnut RM, Ghajar J et al. Guidelines for the management of severe traumatic brain injury. IX. Cerebral perfusion thresholds. J Neurotrauma 2007; 24: (Suppl 1) S59-S64

23 Andrews PJ. Cerebral perfusion pressure and brain ischaemia: can one size fit all? Crit Care 2005; 9: 638-639

24 Alali AS, Fowler RA, Mainprize TG et al. Intracranial pressure monitoring in severe traumatic brain injury: results from the American College of Surgeons Trauma Quality Improvement Program. J Neurotrauma 2013; 30: 1737-1746

25 Kosty JA, Leroux PD, Levine J et al. Brief report: a comparison of clinica and research practices in measuring cerebral perfusion pressure: a literature review and practitioner survey. Anesth Analg 2013; 117: 694-698

26 Steiner LA, Czosnyka M, Piechnik SK et al. Continuous monitoring of cerebrovascular pressure reactivity allows determination of optimal cerebral perfusion pressure in patients with traumatic brain injury. Crit Care Med 2002; 30: 733-738

27 Staykov D, Schwab S. Clearing bloody cerebrospinal fluid: clot lysis, neuroendoscopy and lumbar drainage. Curr Opin Crit Care 2013; 19: 92-100

28 Moradiya $Y$, Murthy SB, Newman-Toker DE et al. Intraventricular thrombolysis in intracerebral hemorrhage requiring ventriculostomy: a decade-long real-world experience. Stroke 2014; 45: 2629-2635

29 Staykov D, Huttner HB, Struffert $T$ et al. Intraventricular fibrinolysis and lumbar drainage for ventricular hemorrhage. Stroke 2009; 40 : 3275-3280

30 Huttner HB, Tognoni E, Bardutzky J et al. Influence of intraventricular fibrinolytic therapy with rt-PA on the long-term outcome of treated patients with spontaneous basal ganglia hemorrhage: a case-control study. Eur J Neurol 2008; 15: 342-349

31 Hanley DF. Intraventricular hemorrhage: severity factor and treatment target in spontaneous intracerebral hemorrhage. Stroke 2009; 40: $1533-1538$

32 Ziai WC, Tuhrim S, Lane $K$ et al. A multicenter, randomized, doubleblinded, placebo-controlled phase III study of Clot Lysis Evaluation of Accelerated Resolution of Intraventricular Hemorrhage (CLEAR III). Int J Stroke 2014; 9: 536-542

33 Steiner LA, Coles JP, Johnston AJ et al. Assessment of cerebrovascular autoregulation in head-injured patients: a validation study. Stroke 2003; 34: 2404-2409

34 Budohoski KP, Czosnyka M, Kirkpatrick PJ et al. Clinical relevance of cerebral autoregulation following subarachnoid haemorrhage. Nat Rev Neurol 2013; 9: 152-163

35 McHedlishvili G. Physiological mechanisms controlling cerebral blood flow. Stroke 1980; 11: 240-248
36 Dagal A, Lam AM. Cerebral autoregulation and anesthesia. Curr Opin Anaesthesiol 2009; 22: 547-552

37 Coles JP, Fryer TD, Coleman MR et al. Hyperventilation following head injury: effect on ischemic burden and cerebral oxidative metabolism. Crit Care Med 2007; 35: 568-578

38 Stocchetti N, Maas AI, Chieregato A et al. Hyperventilation in head injury: a review. Chest 2005; 127: 1812-1827

39 Jaeger $M$, Soehle $M$, Schuhmann $M U$ et al. Clinical significance of impaired cerebrovascular autoregulation after severe aneurysmal subarachnoid hemorrhage. Stroke 2012; 43: 2097-2101

40 Diedler J, Santos E, Poli S et al. Optimal cerebral perfusion pressure in patients with intracerebral hemorrhage: an observational case series. Crit Care 2014; 18: R51

41 Czosnyka M, Smielewski P, Kirkpatrick P et al. Continuous assessment of the cerebral vasomotor reactivity in head injury. Neurosurgery 1997; 41: 11-17 discussion 17-19

42 Aries MJ, Czosnyka M, Budohoski KP et al. Continuous determination of optimal cerebral perfusion pressure in traumatic brain injury. Crit Care Med 2012; 40: 2456-2463

43 Rasulo FA, Girardini A, Lavinio A et al. Are optimal cerebral perfusion pressure and cerebrovascular autoregulation related to long-term outcome in patients with aneurysmal subarachnoid hemorrhage? J Neurosurg Anesthesiol 2012; 24: 3-8

44 Jaeger M, Schuhmann MU, Soehle M et al. Continuous assessment of cerebrovascular autoregulation after traumatic brain injury using brain tissue oxygen pressure reactivity. Crit Care Med 2006; 34: 1783-1788

45 Lazaridis C, Andrews CM. Brain tissue oxygenation, lactate-pyruvate ratio, and cerebrovascular pressure reactivity monitoring in severe traumatic brain injury: systematic review and viewpoint. Neurocrit Care 2014; 21: 345-355

46 Wolf S, Vajkoczy P, Dengler J et al. Drift of the Bowman Hemedex(R) cerebral blood flow monitor between calibration cycles. Acta Neurochir Suppl 2012; 114: 187-190

47 Keller E, Froehlich J, Muroi C et al. Neuromonitoring in intensive care: a new brain tissue probe for combined monitoring of intracranial pressure (ICP) cerebral blood flow (CBF) and oxygenation. Acta Neurochir Suppl 2011; 110: 217-220

48 Keller E, Nadler A, Imhof HG et al. New methods for monitoring cerebral oxygenation and hemodynamics in patients with subarachnoid hemorrhage. Acta Neurochir Suppl 2002; 82: 87-92

49 Martini RP, Deem S, Treggiari MM. Targeting brain tissue oxygenation in traumatic brain injury. Respir Care 2013; 58: 162-172

50 Oddo M, Levine JM, Mackenzie L et al. Brain hypoxia is associated with short-term outcome after severe traumatic brain injury independently of intracranial hypertension and low cerebral perfusion pressure Neurosurgery 2011; 69: 1037-1045 discussion 1045

51 Chen HI, Stiefel MF, Oddo $M$ et al. Detection of cerebral compromise with multimodality monitoring in patients with subarachnoid hemorrhage. Neurosurgery 2011; 69: 53-63 discussion 63

52 Nangunoori R, Maloney-Wilensky E, Stiefel M et al. Brain tissue oxygenbased therapy and outcome after severe traumatic brain injury: a systematic literature review. Neurocrit Care 2012; 17: 131-138

53 Bohman LE, Pisapia JM, Sanborn MR et al. Response of brain oxygen to therapy correlates with long-term outcome after subarachnoid hemorrhage. Neurocrit Care 2013; 19: 320-328

54 Pascual JL, Georgoff P, Maloney-Wilensky E et al. Reduced brain tissue oxygen in traumatic brain injury: are most commonly used interventions successful? J Trauma 2011; 70: 535-546

55 Hecht N, Fiss I, Wolf S et al. Modified flow- and oxygen-related autoregulation indices for continuous monitoring of cerebral autoregulation. J Neurosci Methods 2011; 201: 399-403

56 Dengler J, Frenzel C, Vajkoczy P et al. Cerebral tissue oxygenation measured by two different probes: challenges and interpretation. Intensive Care Med 2011; 37: 1809-1815

57 Winkler MK, Chassidim Y, Lublinsky S et al. Impaired neurovascular coupling to ictal epileptic activity and spreading depolarization in a patient with subarachnoid hemorrhage: possible link to blood-brain barrier dysfunction. Epilepsia 2012; 53 (Suppl 6): 22-30

58 WoitzikJ, Dreier JP, Hecht N et al. Delayed cerebral ischemia and spreading depolarization in absence of angiographic vasospasm after subarachnoid hemorrhage. J Cereb Blood Flow Metab 2012; 32: 203-212

59 Woitzik J, Hecht N, Pinczolits A et al. Propagation of cortical spreading depolarization in the human cortex after malignant stroke. Neurology 2013; 80: 1095-1102

60 Pietrobon D, Moskowitz MA. Chaos and commotion in the wake of cortical spreading depression and spreading depolarizations. Nat Rev Neurosci 2014; 15: 379-393 
61 Hinzman JM, Andaluz N, Shutter LA et al. Inverse neurovascular coupling to cortical spreading depolarizations in severe brain trauma. Brain 2014; 137: 2960-2972

62 Lad SP, Hegen H, Gupta G et al. Proteomic biomarker discovery in cerebrospinal fluid for cerebral vasospasm following subarachnoid hemorrhage. J Stroke Cerebrovasc Dis 2012; 21: 30-41

63 Yokobori S, Hosein K, Burks S et al. Biomarkers for the clinical differential diagnosis in traumatic brain injury - a systematic review. CNS Neurosci Ther 2013; 19: 556-565

64 Hong CM, Tosun C, Kurland DB et al. Biomarkers as outcome predictors in subarachnoid hemorrhage - a systematic review. Biomarkers 2014; 19: 95-108

65 Friedrich $V$, Flores $R$, Muller A et al. Luminal platelet aggregates in functional deficits in parenchymal vessels after subarachnoid hemorrhage. Brain Res 2010; 1354: 179-187

66 Romano JG, Rabinstein AA, Arheart KL et al. Microemboli in aneurysmal subarachnoid hemorrhage. J Neuroimaging 2008; 18: 396-401

67 Schubert GA, Seiz M, Hegewald AA et al. Acute hypoperfusion immediately after subarachnoid hemorrhage: a xenon contrast-enhanced CT study. J Neurotrauma 2009; 26: 2225-2231
68 Goodman JC, Robertson CS. Microdialysis: is it ready for prime time? Curr Opin Crit Care 2009; 15: 110-117

69 Hillered L, Persson L, Nilsson P et al. Continuous monitoring of cerebral metabolism in traumatic brain injury: a focus on cerebral microdialysis. Curr Opin Crit Care 2006; 12: 112-118

70 Hutchinson PJ, O'Connell MT, Rothwell NJ et al. Inflammation in human brain injury: intracerebral concentrations of IL-1alpha, IL-1beta, and their endogenous inhibitor IL-1ra. J Neurotrauma 2007; 24: 15451557

71 Sarrafzadeh A, Copin JC, Bengualid DJ et al. Matrix metalloproteinase-9 concentration in the cerebral extracellular fluid of patients during the acute phase of aneurysmal subarachnoid hemorrhage. Neurol Res 2012; 34: 455-461

72 Larach DB, Kofke WA, Le Roux P. Potential non-hypoxic/ischemic causes of increased cerebral interstitial fluid lactate/pyruvate ratio: a review of available literature. Neurocrit Care 2011; 15: 609-622

73 Timofeev I, Carpenter KL, Nortje J et al. Cerebral extracellular chemistry and outcome following traumatic brain injury: a microdialysis study of 223 patients. Brain 2011; 134: 484-494 